\title{
Follow-up of patients with short-chain acyl-CoA dehydrogenase and isobutyryl-CoA dehydrogenase deficiencies identified through newborn screening: one center's experience
}

\author{
Loren Pena, MD, $\mathrm{PhD}^{1-4}$, Brad Angle, MD¹, Barbara Burton, $\mathrm{MD}^{1}$ and Joel Charrow, MD ${ }^{1}$
}

\begin{abstract}
Purpose: To evaluate the growth, development, and medical histories of children with short-chain acyl-CoA dehydrogenase and isobutyrylCoA dehydrogenase deficiencies identified through newborn screening.

Methods: Chart review of patients diagnosed with short-chain acylCoA dehydrogenase or isobutyryl-CoA dehydrogenase deficiency at our center.

Results: There were 16 children with short-chain acyl-CoA dehydrogenase deficiency, including 10 with two pathogenic mutations, and 8 with isobutyryl-CoA dehydrogenase deficiency. All but one patient has had normal growth and development, and that patient also had the 22q deletion syndrome.
\end{abstract}

Conclusion: Our data and previous reports suggest that the majority of individuals with short-chain acyl-CoA dehydrogenase or isobutyryl-CoA dehydrogenase deficiencies have normal growth and development.

Genet Med 2012:14(3):342-347

Key Words: inborn error of metabolism; isobutyryl-CoA dehydrogenase deficiency; newborn screening; short-chain acyl-CoA dehydrogenase deficiency

\section{INTRODUCTION}

The introduction of tandem mass spectrometry in newborn screening programs has made it possible to quantify the levels of acylcarnitines of varying chain length and thereby diagnose a variety of disorders of organic acid and fatty acid metabolism. As a result, a number of conditions that were previously thought to be rare have been recognized with increasing frequency in otherwise asymptomatic infants, an observation that has raised questions about the clinical significance of the metabolic alterations. Among these are two inherited conditions that result in accumulation of four-carbon carnitine esters (also known as C4 acylcarnitines): short-chain acyl-CoA dehydrogenase (SCAD) deficiency and isobutyryl-CoA dehydrogenase (IBD) deficiency.

SCAD deficiency, a defect in the $\beta$-oxidation of short-chain fatty acids (four to eight carbons in length), was originally reported in 1984 in a 46-year-old woman with lipid myopathy and profound carnitine deficiency, ${ }^{1}$ although she was later thought to have multiple acyl-CoA dehydrogenase deficiency (glutaric aciduria type II). ${ }^{2}$ The first true cases were reported in 1987 in two neonates. ${ }^{3}$ Biochemically, the disorder is characterized by elevated excretion of ethylmalonic and methylsuccinic acids in the urine. SCAD is encoded by the ACADS gene, and a number of mutations have been associated with deficient enzyme activity. ${ }^{4-7}$ Two variants in the ACADS gene, $625 \mathrm{G}>\mathrm{A}$ and $511 \mathrm{C}>\mathrm{T}$, are commonly found in the general population. ${ }^{4,8,9}$ Although these variants are associated with ethylmalonic aciduria and some decreased enzyme activity, they are not thought to cause SCAD deficiency. They may, however, confer disease susceptibility when present with other environmental or genetic factors that remain undetermined. ${ }^{4,10}$

IBD deficiency, a defect in the valine catabolic pathway, was first described in 1998 in a 2-year-old girl with cardiomyopathy and carnitine deficiency whose cardiac function, growth, and development proceeded normally following carnitine supplementation. ${ }^{11}$ IBD is encoded by ACAD $8,{ }^{12}$ and mutations have been found to disrupt its function. ${ }^{11,13}$

Since these initial reports, a number of additional patients with SCAD and IBD deficiencies have been detected and are currently being followed at metabolic centers throughout the world. This report describes the clinical course of children with these two conditions followed at a single center, and suggests that most, if not all, of the children with these conditions detected through newborn screening are healthy and have normal growth and development. 


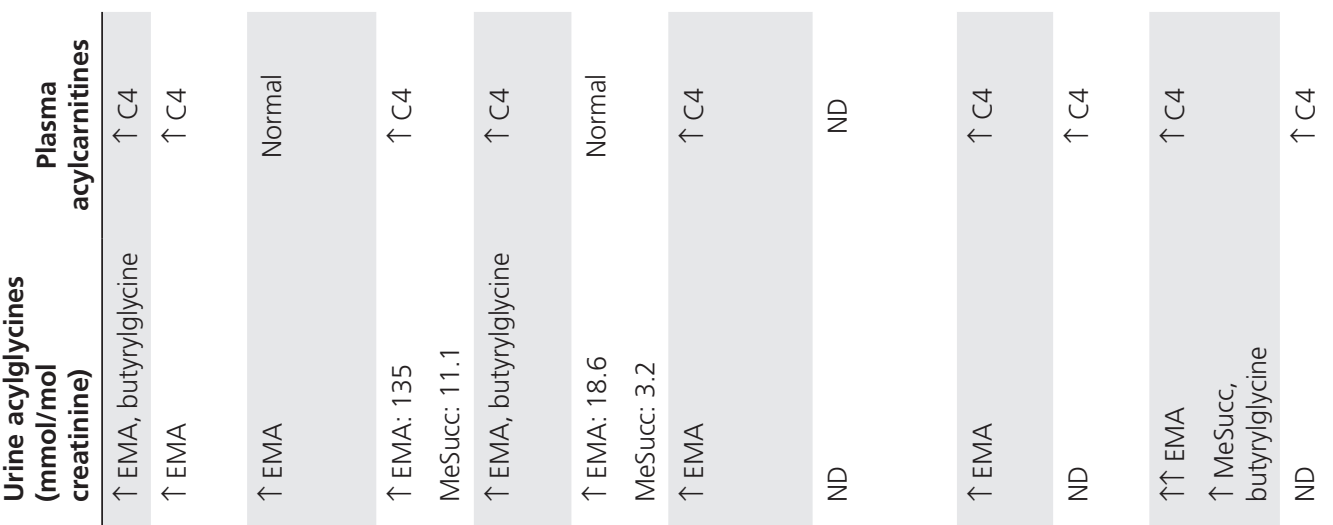

ำ

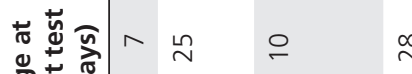

$\stackrel{\infty}{\infty}=$ n

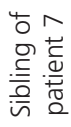

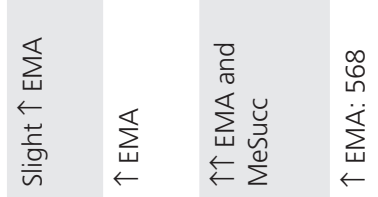

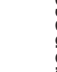

(1)

\section{(1)

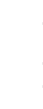

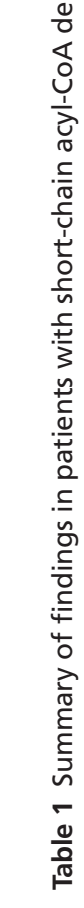




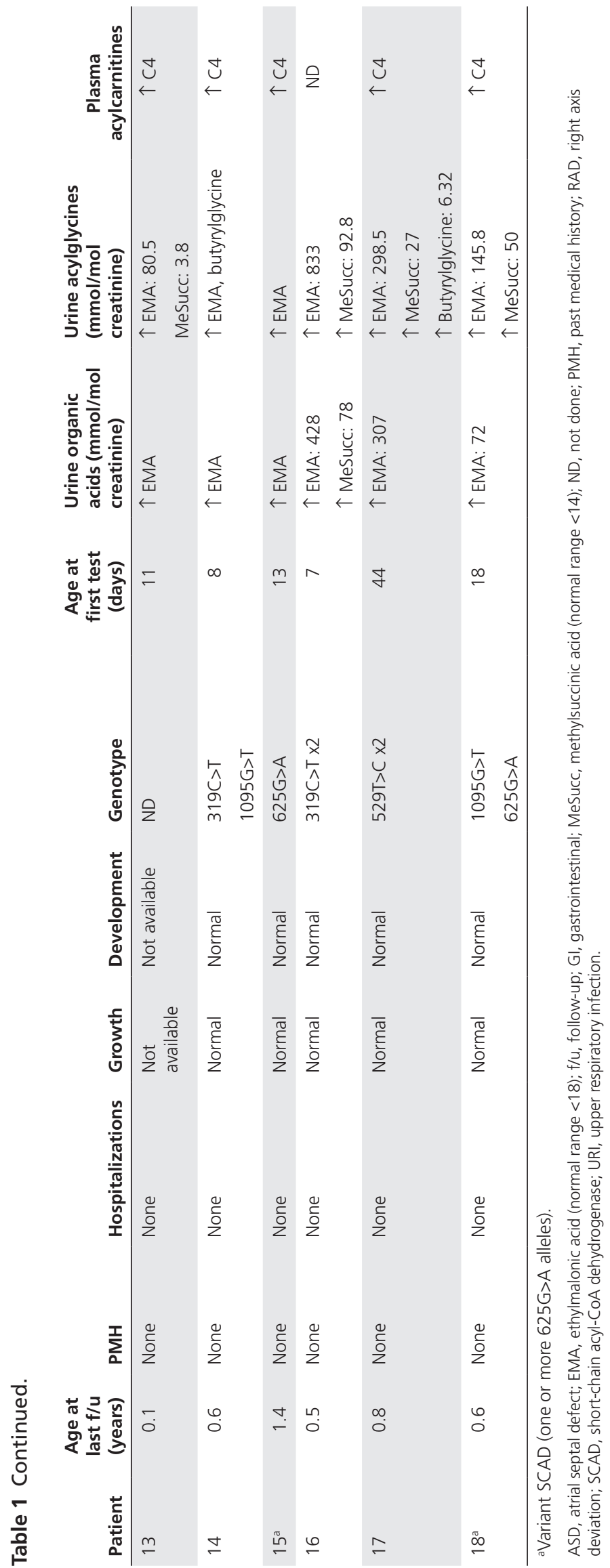




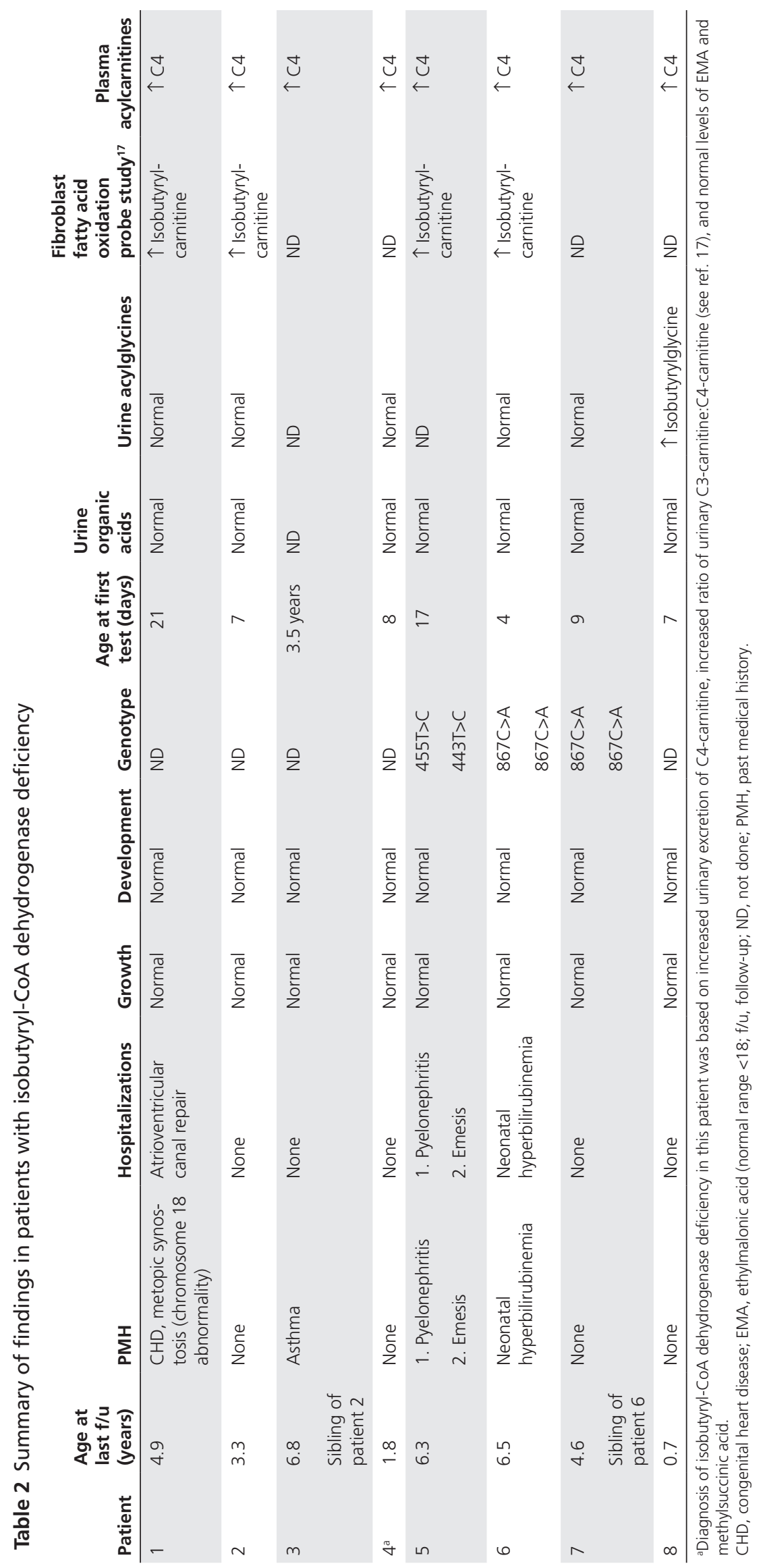




\section{MATERIALS AND METHODS}

The study was approved by the institutional review board at Children's Memorial Hospital in Chicago. We reviewed the charts of all patients diagnosed with SCAD or IBD deficiency who visited the Division of Genetics, Birth Defects and Metabolism at Children's Memorial Hospital since the institution of expanded newborn screening in Illinois in 2002. Extracted data included growth parameters, health and developmental status at the most recent clinic visit, history of major illnesses or hospitalizations, and information pertaining to the patient's biochemical and genetic diagnosis. Information was tabulated without individual patient identifiers and was maintained in a confidential manner.

\section{RESULTS}

Eighteen patients with a diagnosis of SCAD deficiency detected through newborn screening were identified. All had elevations in C4 acylcarnitines and ethylmalonic aciduria. Fifteen patients underwent urine acylglycine analyses: five had elevated butyrylglycine and four had elevated methylsuccinic acid reported. Methylsuccinic acid was reported in the normal range in three patients, and not commented on in eight patients. Full sequencing of ACADS was performed in 16 cases, and two pathogenic mutations were identified in 10 patients. A single pathogenic mutation and variant allele $(625 \mathrm{G}>\mathrm{A})$ ) were found in five patients (patients $3,6,9,10$, and 18; Table 1). One patient was homozygous for the variant allele and had no pathogenic mutations (patient 15). One patient (no. 13) was lost to follow-up shortly after diagnosis; the mean duration of follow-up for the other patients was 2.2 years (range 0.3-5.6 years). All but one of the patients had normal growth and development. The remaining patient (patient 3) had an atrial septal defect and cleft lip and palate. This patient had a deletion in the 22q11 region and was diagnosed with velocardiofacial syndrome. She was hospitalized once with pneumonia, once with gastrointestinal symptoms, and had speech delay.

We have also followed eight patients with IBD deficiency, all but one diagnosed through expanded newborn screening. All had elevated C4 acylcarnitines without ethylmalonic aciduria. Only one of the six patients tested had increased excretion of isobutyrylglycine (Table 2). One patient (no. 3) was diagnosed at 3.5 years of age after a younger sibling tested positive on newborn screening. All of our patients with IBD deficiency have had normal growth and development. After a mean period of follow-up of 4.6 years (range 0.7-6.8 years), we have not observed any complications that may be related to the disorder, with the possible exception of one patient (no. 5) with evidence of carnitine deficiency during a recent hospitalization for gastroenteritis (free carnitine of $15 \mu \mathrm{mol} / \mathrm{l}$ ).

\section{DISCUSSION}

Following the original reports of SCAD deficiency, affected patients have been described with a variety of signs, including hypoglycemia, lactic acidosis, developmental delay, hypotonia, seizures, and cardiomyopathy, with variable responses to treatment and outcomes. ${ }^{3}$ However, recent publications reveal that a number of patients with SCAD deficiency remain asymptomatic.
Developmental and health outcomes for 14 children with SCAD deficiency, 8 of whom were detected through expanded newborn screening, were recently reported. ${ }^{14}$ All eight children identified through newborn screening had normal neuropsychological development and health on follow-up. Of the other six children included in the study, two were normal and four were developmentally abnormal (one with learning disability and three with mental retardation). However, there were supplementary or alternative explanations for the delays in all four children (e.g., prematurity, fetal distress, maternal HELLP syndrome). Furthermore, seven of the eight alleles in these children contained only the variant mutations $(625 \mathrm{G}>\mathrm{A}$ or $511 \mathrm{C}>\mathrm{T})$ and only one allele had a presumably pathogenic mutation $(136 \mathrm{C}>\mathrm{T})$. Waisbren et al. ${ }^{14}$ also refer to an unpublished study of one of the authors, where only 5 of the 44 individuals affected with SCAD deficiency had symptoms of hypoglycemia and hypotonia. Jethva and Ficicioglu ${ }^{15}$ reported their experience with 14 patients with SCAD deficiency followed for 1-7 years. Two patients (siblings) had speech delay, but both parents had "learning disabilities." The other 12 patients were normal.

Fewer than a dozen cases of IBD deficiency have been described in the literature, and the majority of them are doing well, some with carnitine supplementation. For example, the original patient had normal growth and development with carnitine supplementation 4 years after her diagnosis. ${ }^{12}$ Sass et al. ${ }^{13}$ report on two neonates detected through newborn screening, one of whom had normal growth and development at 13 months, and the other of whom had hypotonia and mild developmental delay at 8 months of age. Pedersen et al. ${ }^{16}$ reported another four patients with variable length of follow-up, from 2 to 5 years of age, all of whom had normal growth, although two had speech delay.

We have not observed clinically significant effects of either of these enzyme deficiencies in any of our patients diagnosed through newborn screening. We hypothesize that the illnesses reported in some of the previous cases may have been the result of secondary carnitine deficiency. In other cases, where the enzyme deficiency was diagnosed during the evaluation of a child with neurologic or metabolic alterations, the presence of the enzyme deficiency may have been coincidental. The true relationship between these enzyme deficiencies and clinical outcomes will ultimately be determined only by further observation, as well as by prospective study of patients identified through newborn screening.

\section{DISCLOSURE}

The authors declare no conflict of interest.

\section{REFERENCES}

1. Turnbull DM, Bartlett K, Stevens DL, et al. Short-chain acyl-CoA dehydrogenase deficiency associated with a lipid-storage myopathy and secondary carnitine deficiency. N Engl J Med 1984;311:1232-1236

2. Bhala A, Willi SM, Rinaldo P, Bennett MJ, Schmidt-Sommerfeld E, Hale DE Clinical and biochemical characterization of short-chain acyl-coenzyme A dehydrogenase deficiency. J Pediatr 1995;126:910-915.

3. Amendt BA, Greene C, Sweetman L, et al. Short-chain acyl-coenzyme A dehydrogenase deficiency. Clinical and biochemical studies in two patients. J Clin Invest 1987;79:1303-1309. 
4. Corydon MJ, Vockley J, Rinaldo P, et al. Role of common gene variations in the molecular pathogenesis of short-chain acyl-CoA dehydrogenase deficiency. Pediatr Res 2001;49:18-23.

5. Tein I, Elpeleg O, Ben-Zeev B, et al. Short-chain acyl-CoA dehydrogenase gene mutation (c.319C>T) presents with clinical heterogeneity and is candidate founder mutation in individuals of Ashkenazi Jewish origin. Mol Genet Metab 2008;93:179-189.

6. Bok LA, Vreken P, Wijburg FA, et al. Short-chain Acyl-CoA dehydrogenase deficiency: studies in a large family adding to the complexity of the disorder. Pediatrics 2003;112:1152-1155.

7. Pedersen CB, Kølvraa S, Kølvraa A, et al. The ACADS gene variation spectrum in 114 patients with short-chain acyl-CoA dehydrogenase (SCAD) deficiency is dominated by missense variations leading to protein misfolding at the cellular level. Hum Genet 2008;124:43-56.

8. Nagan N, Kruckeberg KE, Tauscher AL, Bailey KS, Rinaldo P, Matern D. The frequency of short-chain acyl-CoA dehydrogenase gene variants in the US population and correlation with the C(4)-acylcarnitine concentration in newborn blood spots. Mol Genet Metab 2003;78:239-246.

9. van Maldegem BT, Waterham HR, Duran M, et al. The $625 G>A$ SCAD gene variant is common but not associated with increased C4-carnitine in newborn blood spots. J Inherit Metab Dis 2005;28:557-562.

10. Pedersen $C B$, Bross P, Winter VS, et al. Misfolding, degradation, and aggregation of variant proteins. The molecular pathogenesis of short chain acyl-CoA dehydrogenase (SCAD) deficiency. J Biol Chem 2003;278: 47449-47458.

11. Roe CR, Cederbaum SD, Roe DS, Mardach R, Galindo A, Sweetman L. Isolated isobutyryl-CoA dehydrogenase deficiency: an unrecognized defect in human valine metabolism. Mol Genet Metab 1998;65:264-271.

12. Nguyen TV, Andresen BS, Corydon TJ, et al. Identification of isobutyryl-CoA dehydrogenase and its deficiency in humans. Mol Genet Metab 2002;77:68-79.

13. Sass KP, Samder S, Zschocke J. Isobutyryl-CoA dehydrogenase deficiency: Isobutyrylglycinuria and ACAD8 gene mutations in two infants. J Inherit Metab Dis 2004;27:741-745.

14. Waisbren SE, Levy HL, Noble M, et al. Short-chain acyl-CoA dehydrogenase (SCAD) deficiency: an examination of the medical and neurodevelopmental characteristics of 14 cases identified through newborn screening or clinical symptoms. Mol Genet Metab 2008;95:39-45.

15. Jethva R, Ficicioglu C. Clinical outcomes of infants with short-chain acylcoenzyme A dehydrogenase deficiency (SCADD) detected by newborn screening. Mol Genet Metab 2008;95:241-242.

16. Pedersen $C B$, Bischoff $C$, Christensen $E$, et al. Variations in IBD (ACAD8) in children with elevated C4-carnitine detected by tandem mass spectrometry newborn screening. Pediatr Res 2006;60:315-320.

17. Oglesbee D, He M, Majumder N, et al. Development of a newborn screening follow-up algorithm for the diagnosis of isobutyryl-CoA dehydrogenase deficiency. Genet Med 2007;9:108-116. 